\title{
The challenge of supporting care for dementia in primary care
}

\author{
Malaz Boustani ${ }^{1,2,3}$ \\ Cathy Schubert ${ }^{3}$ \\ Youcef Sennour ${ }^{3}$ \\ 'Indiana University Center for Aging \\ Research; ${ }^{2}$ Regenstrief Institute; \\ ${ }^{3}$ Department of Medicine, Indiana \\ University School of Medicine, \\ Indianapolis, Indiana, USA
}

\begin{abstract}
Most patients with dementia receive care within primary care systems and have challenging medical and psychiatric issues. Their dementia related symptoms are often not recognized by the primary care system; they suffer from multiple chronic medical conditions; receive numerous psychotropic medications including anticholinergics; and display clinically relevant behavioral and psychological symptoms. Improving the care for such vulnerable patients demands supporting the primary care system with various resources, including dementia care managers, access to and coordination with interdisciplinary dementia specialists, and a feasible dementia screening and diagnosis process.

Understanding primary care clinics as a complex adaptive system may enhance our capacity to deliver a flexible supportive process using the above crucial resources to adequately assess and effectively manage patients with dementia. Such a complex adaptive system process would have the best probability of surviving the unknowable future challenges that will face the primary care system.
\end{abstract}

A global consensus of dementia epidemiologists estimates that a new patient is diagnosed with dementia every 7 seconds (Ferri et al 2005). In 2000, there were between 3 and 7 million dementia cases in the US (Sloane et al 2002). It is anticipated that, if no scientific advances occur to alter the incidence and progression of dementia, between 8 and 13 million people in the US will be suffering from the syndrome by the year 2050 (Sloane et al 2002). Dementia is an acquired syndrome of decline in memory and at least one other cognitive domain, such as language, visuo-spatial or executive function, sufficient to interfere with social or occupational functioning in an alert person (APA 1994). Alzheimer Disease (AD) is considered the most common type of dementia (APA 1994).

In this manuscript, we first describe the burden of dementia and the current status of dementia care in primary care systems; second, we provide a clinically relevant review of currently available pharmacological interventions to manage the various symptoms of dementia; third, we describe an organizational theory, called complex adaptive system, and its potential application to enhance dementia care within primary care systems; and finally, we provide an example of a recent successful application of this theory.

\section{Dementia burden}

Dementia causes a high burden of suffering for patients and their families (Boustani et al 2003a). For patients, in addition to cognitive and functional deterioration, dementia leads to behavioral and psychological complications, increased use of health and social services, complicated clinical management of other comorbid conditions, and increased risk for medical complications such as delirium, falls, motor vehicle crashes, incontinence, fractures, and infections. For family caregivers, dementia can lead to 
higher levels of anxiety, depression, use of psychotropic medications, sleep problems, and chronic fatigue (Boustani et al 2003a) (see Table 1). In 2003, dementia constituted 11\% of total years lived with disability in people aged 60 years and older. This is more than cancer, stroke or cardiovascular disease (Ferris et al 2005). Currently, Medicare recipients with dementia account for 34\% of Medicare spending, even though they constitute only $13 \%$ of the total Medicare beneficiaries. By 2050, Medicare will be spending over $\$ 1$ trillion on beneficiaries with this syndrome (The Lewin Group, 2004).

\section{The status of dementia care in the primary care system}

The majority of patients with dementia receive their care within a primary care system (Boustani et al 2005; Callahan et al 2006). In this health care setting, dementia prevalence is $2 \%$ among patients aged 65 to 69 years, $7 \%$ among those aged 70 to 79 years, and 17\% among older adults aged 80 and older (Boustani et al 2005). Research studies found that less than a third of these patients are recognized by the primary care system as having dementia (Boustani et al 2003a, 2005). Patients with dementia suffer from numerous chronic medical conditions, receive multiple prescriptions including psychotropic drugs, display a wide range of behavioral and psychological symptoms, and extensively utilize the health care system (Boustani et al 2003a; Callahan et al 2006; Schubert et al 2006) (see Table 2). More than 20\% of these patients with dementia are exposed to at least one definite anticholinergic medication and less than $10 \%$ are

Table I Dementia symptomatology

\begin{tabular}{ll}
\hline Cognitive impairment: & Functional disability: \\
\hline -Memory deficit & -Basic ADL disability \\
-Language deficit & -IADL disability \\
-Executive deficit & \\
-Visuospatial deficit & \\
-Stimulation recognition deficit (agnosia) & \\
Behavioral and psychological & Caregiver burden \\
symptoms: & \\
-Apathy & -Sleep problem \\
-Depression/Dysphoria/Irritability & -Mood problem \\
-Anxiety & -Coping problem \\
-Agitation/Aggression & \\
-Delusions/Hallucinations & \\
-Elation/Euphoria & \\
-Disinhibition & \\
-Aberrant motor behavior & \\
-Sleep disturbance & \\
-Appetite/Eating disorders & \\
\hline
\end{tabular}

Table 2 The status of dementia in primary care (Callahan CM et al 2006 Schubert CC et al 2006).

\begin{tabular}{ll}
\hline Variable & $\%$ or Mean (SD) \\
\hline Mini mental status examination score & $17.5(5.2)$ \\
Number of chronic disease & $2.4(1.40)$ \\
Number of prescribed medications & $5.1(3.8)$ \\
Suffer from behavioral and psychological & $80 \%$ \\
symptoms & \\
Emergency visit or hospitalization within last & $38 \%$ \\
6 months & $<25 \%$ \\
Recognized cases & $<10 \%$ \\
Receiving cholinesterase inhibitors & $>20 \%$ \\
Receiving at least one psychotropic medication & $>20 \%$ \\
Receiving at least one definite anticholinergic & \\
medication & \\
\hline
\end{tabular}

prescribed cholinesterase inhibitors (Schubert et al 2006). Unfortunately, the complicated medical and psychiatric needs of demented patients affect not only their own care but also the health of their informal caregivers (Guerriero Austrom et al 2004; Callahan et al 2006; Schubert et al 2006). Thus, enhancing the primary care of patients with dementia will require additional financial, educational and social resources (Boustani et al 2005; Callahan et al 2006).

\section{Managing the needs of dementia patients in primary care}

Dementia is characterized by a complex interaction of cognitive, functional, behavioral and psychological symptoms that decrease the quality of life for both the patient and the caregiver. Therefore, successful management needs to be composed of interventions for both. The scope of such integrated management must include pharmacological therapies and psychosocial interventions that target the entire spectrum of dementia symptomatology. An efficacious management would most likely stabilize or slow the cognitive decline; stabilize or prevent additional functional disability; and decrease the severity or delay the emergence of behavioral and psychological symptoms of dementia (BPSD) (See Table 3).

\section{Managing cognitive symptoms}

To date, the United States Federal Drug Agency (FDA) and the European Union Drug Evaluation Agency have approved two classes of medications for the treatment of AD, cholinesterase inhibitors (ChEIs) (Donepezil, Rivastigmine, Galantamine) and a NMDA receptor antagonist (Memantine). Recently, the two agencies approved the use of Rivastigmine, a ChEI, for patients with Parkinson Disease Dementia. 
Table 3 Pharmacological management of Alzheimer disease

\begin{tabular}{llll}
\hline Class & Cognition & ADLs & BPSD \\
\hline ChEl & + & + & $+/-$ \\
Memantine & + & + & + \\
Neuroleptics & - & - & $+/-$ \\
Antidepressants & NE & NE & + \\
Anticonvulsants & - & - & $+/-$ \\
\hline
\end{tabular}

- ChEl: Cholinesterase inhibitors; +: significant positive effect; +/-: possible positive effects; - : negative effect; NE: no effect; All of the neuroleptics are not FDA approved for use in Dementia.

ChEIs are generally considered the first line treatment, especially in mild dementia. In comparison to placebo, 6-month therapy with a ChEI has demonstrated statistically significant positive effects on cognition (Boustani, $\mathrm{M}$ et al 2003a; 2003b). The level of improvement shown was approximately equivalent to a 5 - to 10 -month delay in the progression of the disease. Memantine is approved for the treatment of patients with moderate to severe $A D$ (Mini Mental Status Examination $<18$ ). In comparison to placebo, Memantine produces a similar effect to ChEIs on patients' cognition with a mean difference of 4 points on the Severe Impairment Battery (SIB) (SIB range is 0 to 100 points) (Areosa Sastre A et al 2006). Moreover, Memantine has also been evaluated as an add-on (combined) therapy to ChEIs among patients with moderate to severe dementia and has led to a significant cognitive difference of 3 points on the SIB (Areosa Sastre et al 2006). Treatment with ChEIs and Memantine may, however, lead to adverse effects such as nausea, vomiting, and diarrhea for ChEIs and headache, confusion and constipation for Memantine (Boustani M et al 2003a, 2003b; Areosa Sastre A et al 2006).

\section{Managing functional disability}

Since there is a lack of universally accepted measures to evaluate the impact of ChEIs and Memantine on the functional performance of patients with $\mathrm{AD}$ and other dementias, it is difficult to appreciate the day to day impact of these medications on functional disability. In general, the effect of ChEI and Memantine on daily function appears minimal (Boustani et al 2003a, 2003b; Livingston and Katona 2004; Areosa Sastre et al 2006). However, using time to functional decline as an outcome measure and in comparison to placebo, Donepezil demonstrated a 5-month delay in such a decline (Boustani et al 2003a, 2003b). Furthermore, 34\% of patients with moderate to severe AD did not decline functionally after 6 months of treatment with Memantine versus $20 \%$ of those receiving placebo (Livingston and Katona 2004).

\section{Managing behavioral and psychological symptoms of dementia (BPSD)}

The non-cognitive behavioral and psychological symptoms in dementia (BPSD) can be defined as verbal, vocal, or motor behavior that is inappropriate for the setting, and reflects inadequate ability to cope with internal and/or environmental stressors. BPSD includes a heterogeneous range of psychological reactions, psychiatric symptoms, and behaviors. A variety of both internal and external stressors can be involved. More than $90 \%$ of patients with dementia will experience BPSD at some point during the course of their illness (Finkel et al 2000). Aggression is the most serious symptom and seems to be present in $30 \%$ of patients with dementia attending primary care clinics (Haupt et al 2000).

The current management of aggression is, at best, moderately successful, even in controlled trials. One reason for the limited effectiveness of aggression management is the current focus on pharmacologic interventions used in the setting of an acute crisis. The traditional pharmacological management of BPSD in general and aggression in particular includes the use of typical and atypical neuroleptics, antidepressants, anticonvulsants, hypnotics, and ChEIs. However, these pharmacologic interventions have not been approved by the FDA for such therapy, their efficacy is limited, and they produce numerous side effects (Schneider et al 2005; Sink et al 2005; Schneider et al 2006). Neuroleptics in particular can lead to the development of extra-pyramidal signs, gait abnormalities, sedation, increased incidence of cerebrovascular events, and higher mortality rates (Schneider et al 2005, 2006; Sink et al 2005).

\section{Building a complex adaptive primary care system for dementia}

Complex adaptive system (CAS) is an open, dynamic and flexible network that is considered complex due to its composition of numerous interconnected, semi-autonomous, competing and collaborating members (McDaniel et al 2003; Stoebel et al 2005). The members of CAS can be individuals, teams, functional groups, social institutions, or organizational processes. The CAS is considered adaptive due to its capability of learning from its previous experiences and its flexibility to accommodate change in the connecting pattern of its members (McDaniel et al 2003; Stoebel et al 2005). CAS is characterized (see Table 4) by emergent behaviors as opposed to predetermined ones and self-organized control instead of hierarchical pyramid-based central control (McDaniel et al 2003; Stoebel et al 2005). 
Table 4 Key characteristics of complex adaptive primary care system

\begin{tabular}{ll}
\hline Members/Agents & $\begin{array}{l}\text { Semi-autonomous clinicians, } \\
\text { administrators, clerks, and patients who } \\
\text { have the capacity to exchange information } \\
\text { with other individuals and with their } \\
\text { surrounding environment and subsequently } \\
\text { change their behaviors. } \\
\text { The system members interact locally in } \\
\text { nonlinear pattern that lead to rich } \\
\text { relationships and network of interrelated } \\
\text { interconnections/ } \\
\text { Relationships } \\
\text { A process that allows the system's } \\
\text { members to mutually adjust their } \\
\text { behaviors to cope with changing internal } \\
\text { and external environmental demands. Results } \\
\text { in a new structures and patterns over time } \\
\text { The system self-organizing process may } \\
\text { induce the birth of new system properties, } \\
\text { features, patterns, and relationships that } \\
\text { are distinct from the members' identities, } \\
\text { properties or features } \\
\text { A process of reciprocal makeover of both } \\
\text { the system members and their surrounding } \\
\text { environment that leads to the creation of } \\
\text { interdependency of the present and the past } \\
\text { system's experiences. }\end{array}$ \\
\hline
\end{tabular}

Health care delivery organizations such as primary care clinics are an example of CAS (Stroebel et al 2005). These clinics usually include administrative assistants, clinic managers, nursing staff, clinical providers, billing specialists and other individuals who each bring their own perspectives, skills, experiences, and goals to patient care. These clinic members interact constantly in a nonlinear way and are continually faced with external and internal changes, such as patient's medical status, insurance requirements, regulations, new research findings, member turnover and legal issues. Primary care clinics have been described as complex adaptive systems with local critical nonlinear relationships that produce unpredictable behavioral patterns or dynamics (McDaniel et al 2003; Stoebel et al 2005). Any attempt to rigidly control this CAS may increase problems by paralyzing the members' ability to learn and take action to affect outcomes (McDaniel et al 2003; Stoebel et al 2005). Although the current system of primary care does not have the resources to provide the appropriate diagnosis, evaluation, education, and complex management required for patients with dementia (Gueerriero Austrom et al 2004; Boustani et al 2005; Callahan et al 2006; Schubert et al 2006), understanding primary care clinics as a CAS may enhance our capacity to develop a flexible process of minimally standardized approaches to assess and manage patients with dementia. Such a process would have the best probability of surviving the unknowable future challenges that will face these primary care clinics. Over the past decade, Indiana University Center for Aging Research used the lens of CAS to develop such a collaborative and minimally standardized approach to assess and manage patients with dementia within a primary care system called PREVENT-Memory Loss (Boustani et al 2005; Callahan et al 2006; Guerriero Austrom et al 2004). The efficacy of PREVENT program was evaluated in a randomized controlled trial that enrolled 153 older adults with Alzheimer disease and included their caregivers (Callahan et al 2006).

The PREVENT program included the following minimally specified components: (1) dementia care manager (DCM); (2) electronic tracking of patients status; (3) interdisciplinary dementia management resources; (4) collaborative work with the patients' primary care provider; and (5) caregiver support (see Figure 1). The patients were enrolled in PREVENT via a screening and diagnosis program for all patients aged 65 and older presenting to their primary care physician with or without dementia related symptoms. The DCM provided each dyad of patient and caregiver with educational material related to the nature of Alzheimer disease and community resources. This DCM trained the patient's caregiver in problem solving skills related to patients' functional disability and behavioral and psychological symptoms; the DCM was also available to the caregivers over the phone or for face-to-face visits at the primary care clinic or the patient's home for any dementia related crisis, such as aggressive behaviors and caregiver burden. Moreover, the DCM coordinated a monthly caregiver support meeting that included an exercise program for the patients. In addition to consideration for enhancing the patient's cholinergic system via stopping any definite anticholinergic medication and initiating treatment with cholinesterase inhibitors, the PREVENT interdisciplinary resource team developed standard pharmacological and non-pharmacological protocols to identify, monitor, and treat behavioral and psychological symptoms of dementia (Guerriero Austrom et al 2004). In comparison to patients randomized to the usual care, PREVENT patients had significantly fewer BPSD ( $43 \%$ vs $71 \%$ with $\mathrm{P}<0.05)$ (Callahan et al 2006). Furthermore, PREVENT caregivers reported significant improvements in their stress and depressive symptoms. Such improvement in BPSD was not related to higher use of neuroleptics $(13 \%$ vs $7 \%$, $\mathrm{p}=0.3)$ or sedatives $(10 \%$ vs $9.5 \%, \mathrm{p}=1.0)$. However, 


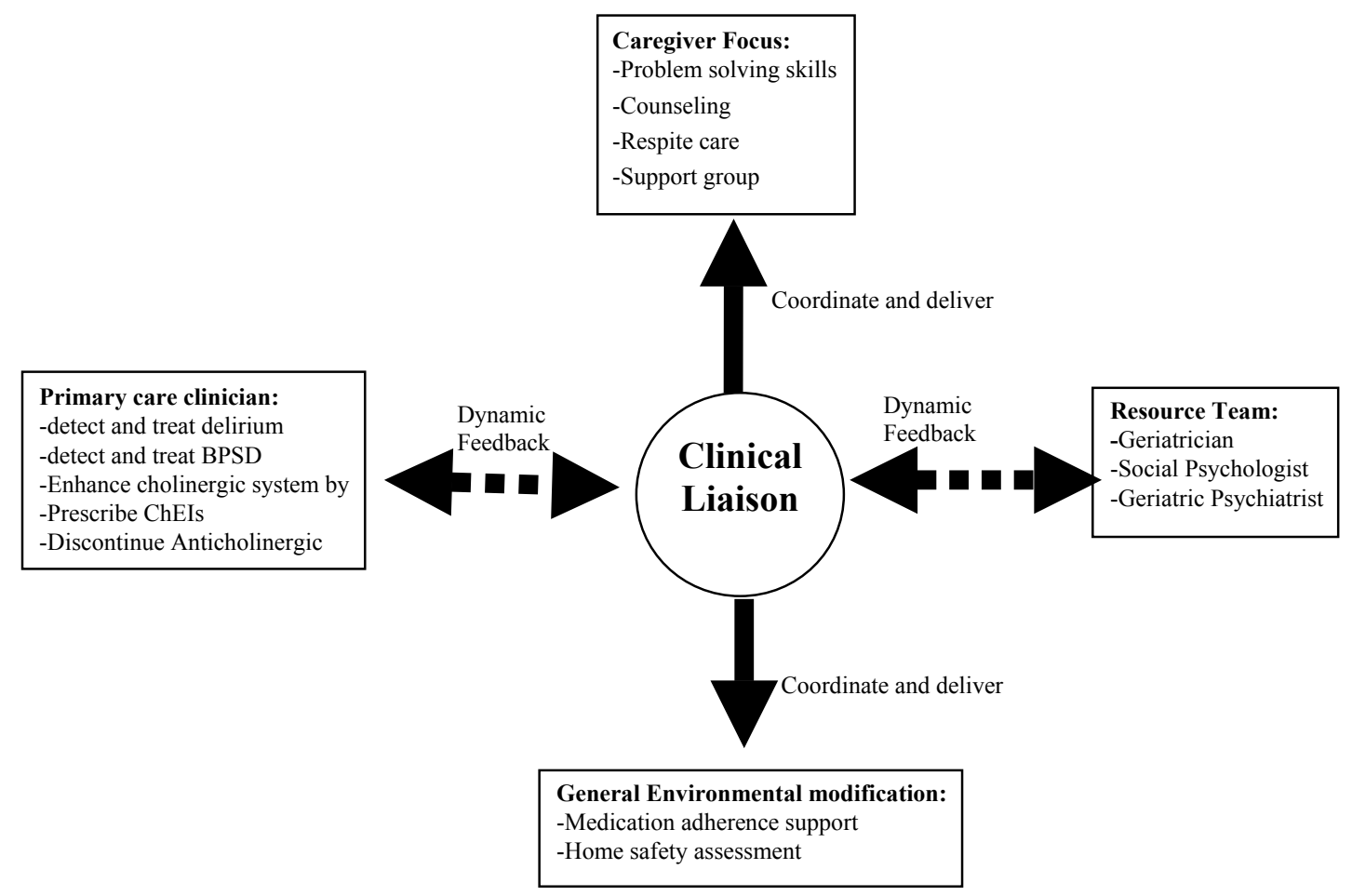

Figure I The Outline for PREVENT program in primary care.

PREVENT patients were more likely to receive ChEIs $(80 \%$ vs $55 \%, \mathrm{p}<0.01)$ and antidepressants $(45 \%$ vs $28 \%$, $\mathrm{p}<0.05)$. Despite its impact on BPSD and caregiver stress, PREVENT program did not affect the patients' cognition, activities of daily living, or rates of hospitalization, nursing home placement or death.

\section{Summary and conclusion}

The management of patients with dementia in primary care is a challenge to our current health care delivery. Redesigning our health care system using the lens of CAS to accommodate a program such as PREVENT would address this challenge. Healthcare delivery programs such as PREVENT enhance the current primary care system by integrating a dementia recognition process within a dementia management system. To succeed, this program must be coordinated by a clinician liaison trained in dementia management and supported with access to community resources and interdisciplinary specialists in dementia care, such as geriatricians, geriatric psychiatrists, geriatric neurologists and geriatric psychologists.

\section{Supported by}

Dr. Boustani was supported by the Paul B. Beeson K23

Career Development Award \# 1-K23-AG026770-01.

\section{References}

[APA] American Psychiatric Association. 1994. Diagnostic and statistical manual of mental disorders, 4th ed. Washington, DC: American Psychiatric Association.

Areosa Sastre A, Sherriff F, McShane R. 2006. Memantine for dementia, Cochrane Dementia and Cognitive Improvement Group, Cochrane Database of Systematic Reviews.

Boustani M, Peterson B, Hanson L, et al. 2003a. Screening for dementia. Systematic evidence review. URL: http://www.ahrq.gov/clinic/uspstfix. $\mathrm{htm}$. Agency for Healthcare Research and Quality, Rockville, MD.

Boustani M, Peterson B, Hanson L, et al. 2003b. Screening for dementia in primary care: a summary of the evidence for the U.S. Preventative Services Task Force. Annals of Internal Medicine, 138:927-37.

Boustani M, Callahan CM, Unverzagt FW, et al. 2005. Implementing a screening and diagnosis program for dementia in primary care. Journal of General Internal Medicine, 20:572-7.

Callahan CM, Boustani MA, Unverzagt FW, et al. 2006. Effectiveness of collaborative care for older adults with Alzheimer disease in primary care: a randomized controlled trial. Journal of American Medical Association, 295:2148-57.

Ferri CP, Prince M, Brayne C, et al. 2005. Global prevalence of dementia: a Delphi consensus study. Lancet, 366:2112-17.

Finkel SI, Burns A, Cohen GD. 2000. Overview. International Psychogeriatrics, 12(Suppl):13-18.

Guerriero Austrom M, Damush TM, Hartwell CW, et al. 2004. Development and implementation of nonpharmacologic protocols for the management of patients with Alzheimer's disease and their families in a multiracial primary care setting. Gerontologist, 44:548-53.

Haupt M, Kurz A, Janner M. 2000. A 2-year follow-up of behavioural and psychological symptoms in Alzheimer's disease. Dementia Geriatrics Cognitive Disorders, 11:147-52.

The Lewin Group. 2004. Saving lives. Saving money: dividends for Americans investing in Alzheimer research. Report of the Lewin Group to the Alzheimer's Association. 
Livingston G, Katona C. 2004. The place of memantine in the treatment of Alzheimer's disease: a number needed to treat analysis. International Journal of Geriatric Psychiatry, 19:919-25.

McDaniel RR Jr, Jordan ME, Fleeman BF. 2003. Surprise, surprise, surprise! A complexity science view of the unexpected. Health Care Management Review, 28:266-78.

Schneider LS, Dagerman KS, Insel P. 2005. Risk of death with atypical antipsychotic drug treatment for dementia: meta-analysis of randomized placebo-controlled trials. Journal of American Medical Association, 294:1934-43.

Schneider LS, Tariot PN, Dagerman KS, et al. 2006. Effectiveness of atypical antipsychotic drugs in patients with Alzheimer's disease. New England Journal of Medicine, 355:1525-38.
Schubert CC, Boustani M, Callahan CM, et al. 2006. Comorbidity profile of dementia patients in primary care: are they sicker? Journal of American Geriatric Society, 54:104-9.

Sink KM, Holden KF, Yaffe K. 2005. Pharmacological treatment of neuropsychiatric symptoms in dementia: a review of the evidence. Journal of American Medical Association, 293:596-608.

Sloane PD, Zimmerman S, Suchindran C, et al. 2002. The public health impact of Alzheimer's disease, 2000-2050: potential implication of treatment advances. Annual Review of Public Health, 23:213-31.

Stroebel CK, McDaniel RR Jr, Crabtree BF, et al. 2005. How complexity science can inform a reflective process for improvement in primary care practices. Joint Commission Journal on Quality Patient Safety, $31: 438-46$. 